\title{
Observations of transient high temperature vortical microstructures in solids during adiabatic shear banding
}

\author{
P. R. Guduru, G. Ravichandran, and A. J. Rosakis* \\ Graduate Aeronautical Laboratories, 105-50 California Institute of Technology, Pasadena, California 91125
}

(Received 28 February 2001; published 30 August 2001)

\begin{abstract}
By using a unique infrared high-speed camera especially constructed for recording highly transient temperature fields at the microscale, we are able to reveal the spatial and temporal microstructure within dynamically growing shear bands in metals. It is found that this structure is highly nonuniform and possesses a transient, short range periodicity in the direction of shear band growth in the form of an array of intense "hot spots" reminiscent of the well-known, shear-induced hydrodynamic instabilities in fluids. This is contrary to the prevailing classical view that describes the deformations and the temperatures within shear bands as being essentially one-dimensional fields. These observations are also reminiscent of the nonuniform structure of localized shear regions believed to exist, at an entirely different length scale, in the earth's lower crust and upper mantle.
\end{abstract}

DOI: 10.1103/PhysRevE.64.036128

PACS number(s): 05.70.-a

When a solid is loaded, it initially undergoes elastic deformation. As the applied load exceeds a certain limit and if the solid is brittle, like glass, it breaks into two or more pieces through the propagation of a network of discrete cracks. However, if the solid is ductile, like a metal, it undergoes plastic (irreversible or permanent) deformation wherever the local stresses exceed the material elastic limit. This deformation is often stable in the sense that if the load is increased gradually, plastic deformation also accumulates gradually. However a dramatically different mode of deformation is also observed in ductile solids when these solids are loaded very rapidly, a situation usually encountered in impact, fragmentation, armour penetration, etc. Intense and unstable shear plastic deformation is localized along very narrow regions in which the temperature approaches the material melting temperature, sometimes leading to a solid-solid or solid-liquid phase transformation. These bands are commonly known as dynamic shear bands. An example of such a band is shown in Fig. 1(c). During the formation of these shear bands, most of the solid experiences very little plastic deformation whereas the bands themselves experience very high plastic strains, typically $10-10^{2}$, occurring at very high rates of $10^{5}-10^{7} \mathrm{~s}^{-1}$. Usually, these bands are tens to hundreds of microns in width. When shear bands are formed under dynamic loading, there is also rapid increase in local temperature in a very short duration, a few microseconds, which means that the local heat generation rate is much larger than the hear conduction rate to the surrounding material. Thus, the dynamic deformation process in the localizing region is supposed to be approximately adiabatic and hence this phenomenon, described by Clifton[1], is also called adiabatic shear banding.

Adiabatic shear bands are coupled thermomechanical instabilities of plastic deformation in solids and the commonly accepted explanation for their formation is as follows. When solids experience dynamic plastic deformation, most of the

\footnotetext{
*Corresponding author.

Electronic address: rosakis@atlantis.caltech.edu
}

external work done is dissipated as heat, which raises the local temperature of the material [2]. When a slightly inhomogeneous plastic deformation occurs in an otherwise uniformly deforming material due to the presence of a geometric discontinuity or a small material inhomogeneity, and if the deformation is rapid enough to supersede heat conduction effects, local temperature is elevated at those locations of inhomogeneities. This increase in temperature softens the material locally and reduces its resistance to further plastic deformation, i.e., local yield stress is lowered. As a result, plastic deformation becomes more inhomogeneous, leading to a self-sustaining cycle of instability: inhomogeneous shear plastic flow $\rightarrow$ nonuniform temperature distribution $\rightarrow$ local thermal softening $\rightarrow$ further inhomogeneous shear plastic flow. The result is the formation of a highly localized adiabatic shear band, starting from a small nonuniformity of deformation. Clearly, any factors that oppose thermal softening

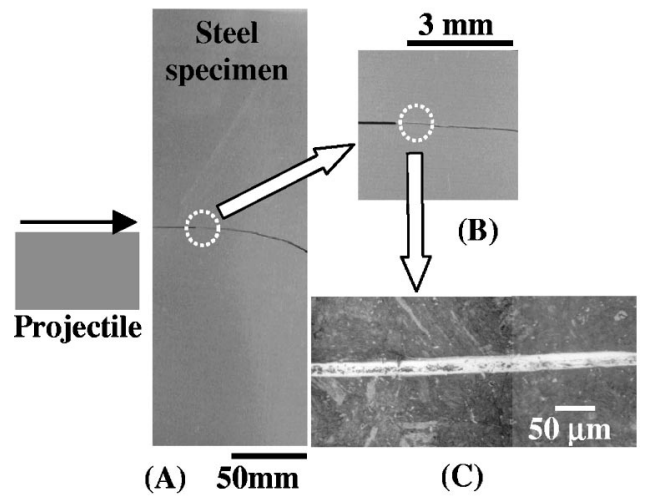

FIG. 1. (a) An illustration of specimen geometry and impact arrangement. The thickness of the plate is $12.5 \mathrm{~mm}$. The steel projectile impacts below the notch, resulting in the formation of a shear band that propagates across the width of the specimen. (b) An area near the crack tip is magnified. (c) A scanning electron microscope image of the shear band. The portion of the shear band where the temperature increase was high enough to cause a phase transformation can be seen as a white band. 
limit the tendency towards shear localization. For example, if the material is thermally very conductive, such as copper, the extra heat generated locally at an inhomogeneity is quickly conducted away, resulting in no thermal softening. Plastic deformation hardens some solids (strain hardening) and this increased resistance to plastic flow opposes the decrease due to thermal softening and renders the formation of adiabatic shear bands more difficult. Increase in strength of the material due to high strain rate also has similar influence (strain rate hardening) $[1,3]$.

The question of shear localization is also of central importance in geophysics. Indeed, at an entirely different length scale, localized shear regions are observed in the earth's lower crust and upper mantle induced by tectonic deformations. The nature of shear localization in this setting has been studied in detail by Poirier [4]. Brun \& Cobbold [5], and Evans and Wong [6]. Shear bands often act as crack initiation and growth sites in structural materials and possible sites of shear rupture in geophysical settings. Indeed, they offer potentially weak paths for crack propagation, thus undermining the strength of the solid and the integrity of structures [3]. Owing to their fundamental scientific significance as well as their technological implications, a number of models have been proposed to predict the critical conditions for their initiation and to model their structure after initiation [7-15]. However, there have been very few experimental attempts to determine the detailed two dimensional structure of initiating and propagating shear bands, mainly due to substantial experimental difficulties involving limited spatial and temporal resolution of the available measurement techniques. Marchand and Duffy [16] and Zhou, Rosakis, and Ravichandran [17] employed linear arrays of 8 and 16 infrared (IR) temperature detectors, respectively, to measure temperature at discrete points along a line across a propagating shear band. However, shear bands are two-dimensional entities, with a well-defined and rapidly propagating tip and an evolving temperature structure behind it that carries information about energy dissipation. Since these measurements were one dimensional in nature, they naturally failed to reveal any information about the two-dimensional (2D) structure of advancing shear bands.

In order to transcend these shortcomings and to perform a detailed transient, real time, high speed, microscopic, and full field imaging of the two-dimensional structure of propagating shear bands, we have designed and constructed a unique infrared high-speed microscopic camera. Since the events we are trying to observe occur over a few microseconds, we designed our thermal camera to capture images at a rate of $10^{6} \mathrm{~s}^{-1}$ with a response time of $500 \mathrm{~ns}$. The camera shown here in Fig. 2(a) features a square array of 64 detectors in an $8 \times 8$ format [see Fig. 2(b)]. Each detector is $100 \times 100 \mu \mathrm{m}^{2}$ in size and is separated by a distance of 30 $\mu \mathrm{m}$ from its neighbor. The detectors are operated at a temperature of $77 \mathrm{~K}$ to maximize the signal to noise ratio. A detailed description of the camera, its construction, and characteristics are reported elsewhere [18]. The distinguishing features of this new IR imaging system are its extremely fast response, a high rate of image acquisition, and its 2D imaging capability at the microscale. The existing and commer-

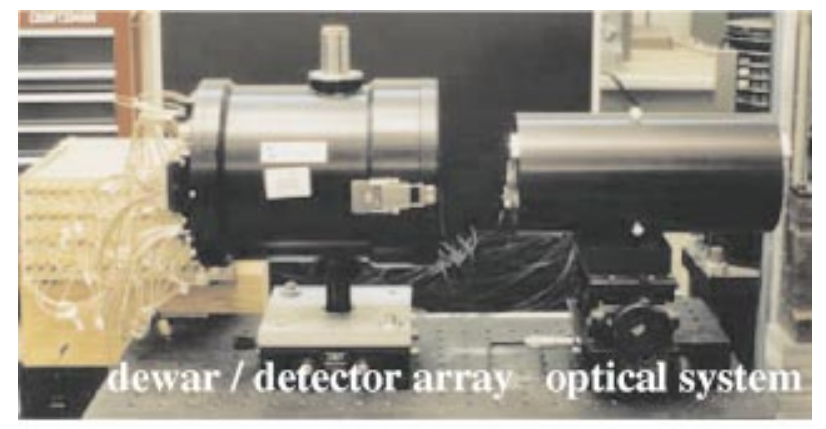

(A)

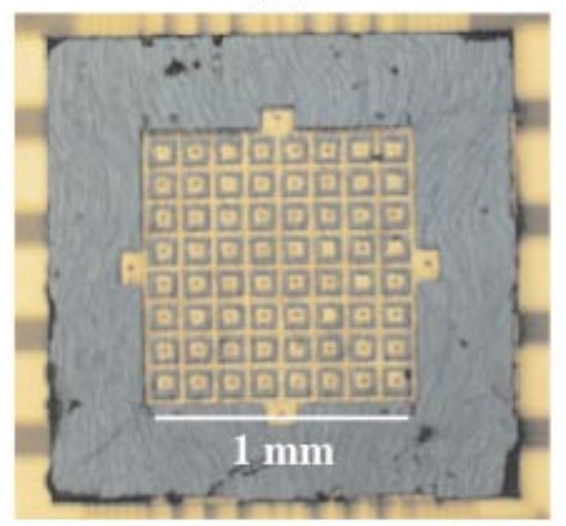

(B)

FIG. 2. (Color) A photograph of the high-speed infrared microscopic camera used in imaging the propagating shear bands. The system features a square array of $64 \mathrm{HgCdTe} I R$ detectors, shown in (b), which is housed in a liquid nitrogen cooled dewar shown in (a). A double Schwarzchild optical imaging system collects the IR radiation emitted by the specimen and focuses it on the detector array. The 64 detector signals are then amplified and recorded simultaneously.

cially available $2 \mathrm{D}$ infrared imaging systems are highly inadequate for the current investigation since they operate at a maximum speed of only a few thousand frames per second and their response time is typically several tens of microseconds. Perhaps the most important feature that makes our camera truly unique is its temporally frozen image acquisition capability. Unlike the existing imaging systems, signals from all detectors are read at the same instant of time, obviating scanning delays. Such a requirement is mandated by the extremely short time scales of shear band initiation and propagation.

To accurately study the two-dimensional nature of adiabatic shear bands, a specimen geometry and a loading arrangement that provide highly controlled shear band initiation and propagation are required. The setup used in the current investigation is illustrated in Fig. 3. The specimen is made of a high strength martensitic steel (C 300). A notch is cut in it, as shown in the figure, using electric discharge machining. The specimen is then subjected to fatigue loading to introduce a sharp 2-mm-long crack at the tip of the notch. The dynamic loading is provided by impacting the specimen edge under the notch with a steel projectile, as shown in Fig. 3 . The impact produces an intense and rapid shear deforma- 


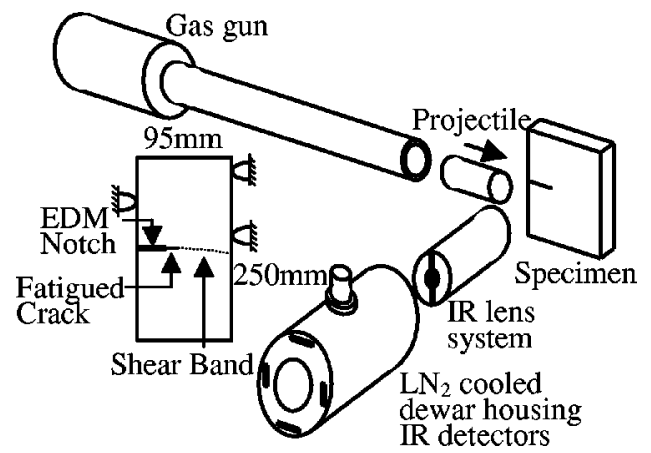

FIG. 3. A schematic illustration of the experimental setup. The specimen has a notch made by Electric Discharge Machining (EDM) that is further extended by fatiguing to introduce a sharp crack. The specimen is supported as shown. A gas gun fires a projectile that impacts the specimen edge below the notch. A double Schwarzchild infrared (IR) telescope collects part of the IR radiation emitted by a small area $\left(1.1 \times 1.1 \mathrm{~mm}^{2}\right)$ along the path of the advancing shear band and focuses it on the IR detector plane housed in a liquid nitrogen $\left(\mathrm{LN}_{2}\right)$ cooled dewar.

tion at the tip of the fatigued crack $[17,19-21]$. When the impact speed exceeds $30 \mathrm{~m} / \mathrm{s}$, a shear band initiates at the crack tip and then propagates across the specimen. Figure 1(a) shows a shear band that propagated across the entire specimen width. Figure 1(c) is a scanning electron microscope image of the shear band, where the region that experienced a phase transformation can be seen as a white band.
The first goal of this investigation was to capture the initiation of the shear band by using the newly developed highspeed IR microscopic camera. The specimen was impacted at a speed of $33 \mathrm{~m} / \mathrm{s}$ while the IR camera was focused at the fatigued crack tip. Each detector measures the average temperature over an area of $110 \times 110 \mu \mathrm{m}^{2}$ on the specimen. Since the width of the shear band is smaller than this, the temperatures reported here are lower bound estimates of the actual temperatures. Figure 4 shows a sequence of selected images illustrating the development of initial temperature field at the tip of the fatigued crack. A plastic zone builds up rapidly up to $20 \mu \mathrm{s}$, with a maximum temperature rise $\Delta T$ of at least $80 \mathrm{~K}$. Subsequently, the images show a band shooting forward. This indicates the transition of the plastic zone in to a growing shear band by localization of deformation. This is the first two-dimensional real time visualization of the highly transient temperature field associated with dynamic shear band initiation.

In order to visualize the propagating tip of a shear band, the IR camera was focused at a distance of $3 \mathrm{~mm}$ in front of the fatigued crack tip in an otherwise identical experiment. Upon impact, the shear band initiated and propagated across the field of view, enabling the camera to capture its structure. Figure 5 shows a sequence of images revealing the structure of the shear band tip as it traverses across the field of view at an average speed estimated to be $500 \mathrm{~m} / \mathrm{s}$. For the first time, these high-speed images capture the details of the dynamically propagating shear band process zone, i.e., the region in
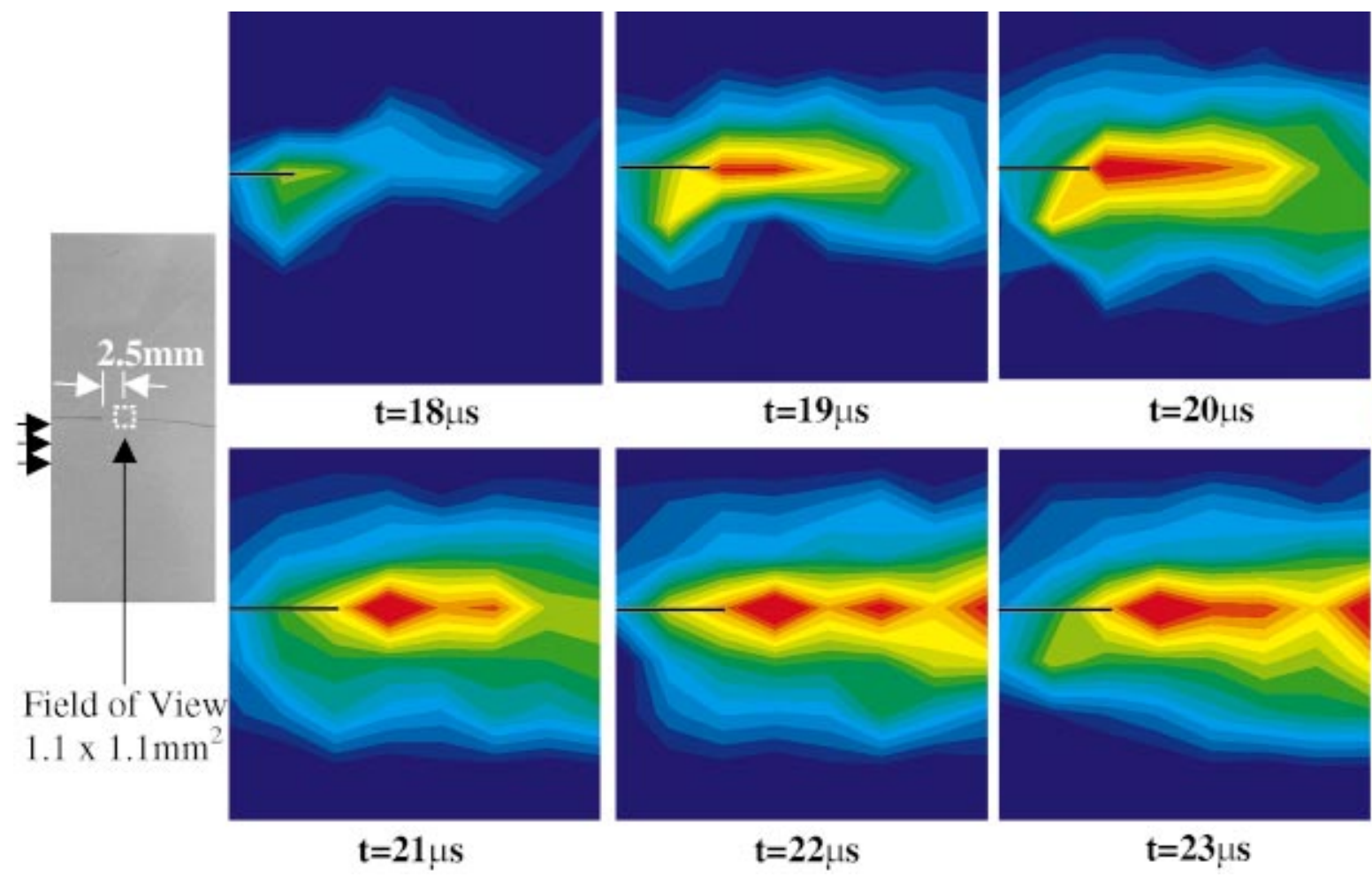

$\Delta \mathbf{T}(\mathbf{K})$
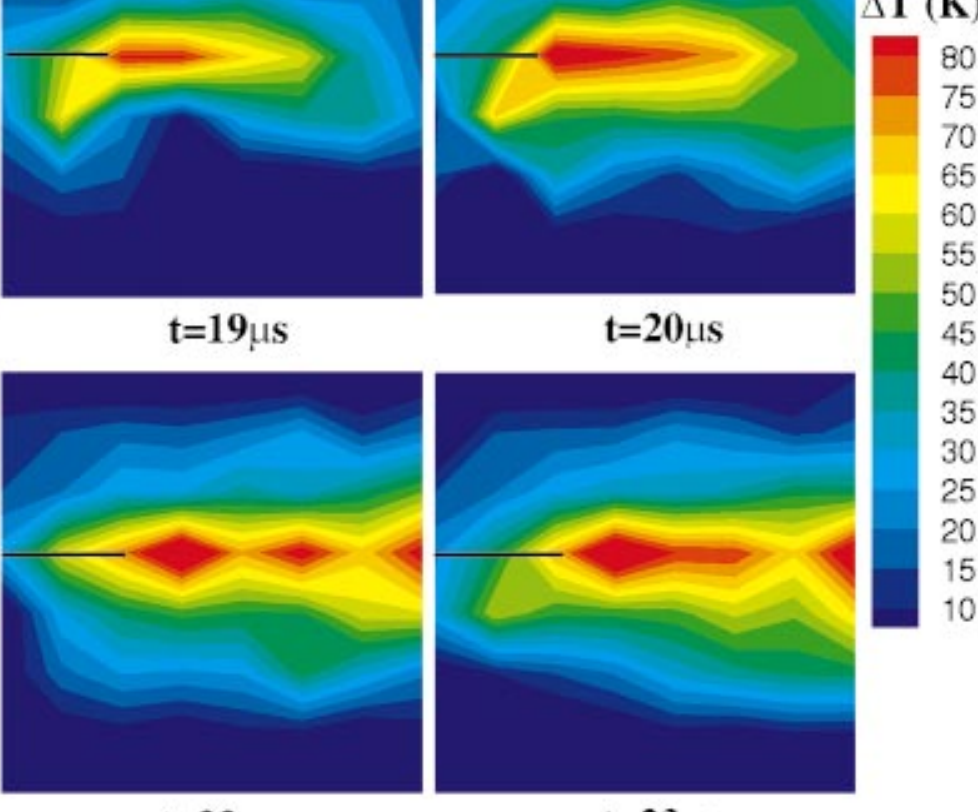

75
70

65

60

55

50

45

40

35

30

25

20

15

10

FIG. 4. (Color) A sequence of high-speed thermal images of the crack tip region in an experiment where the impact speed was $33 \mathrm{~m} / \mathrm{s}$. A plastic zone, corresponding to the dynamic shear loading, rapidly builds up until $20 \mu$ s. Beyond $20 \mu$ s, the plastic zone becomes unstable and a transition to a shear band follows. This transition is captured in the last three images of the above sequence. The temperature rise at the core of the plastic zone when the transition occurs is at least $80 \mathrm{~K}$. The approximate location of the fatigued crack is indicated by the dark lines that are drawn on the images for clarity. 


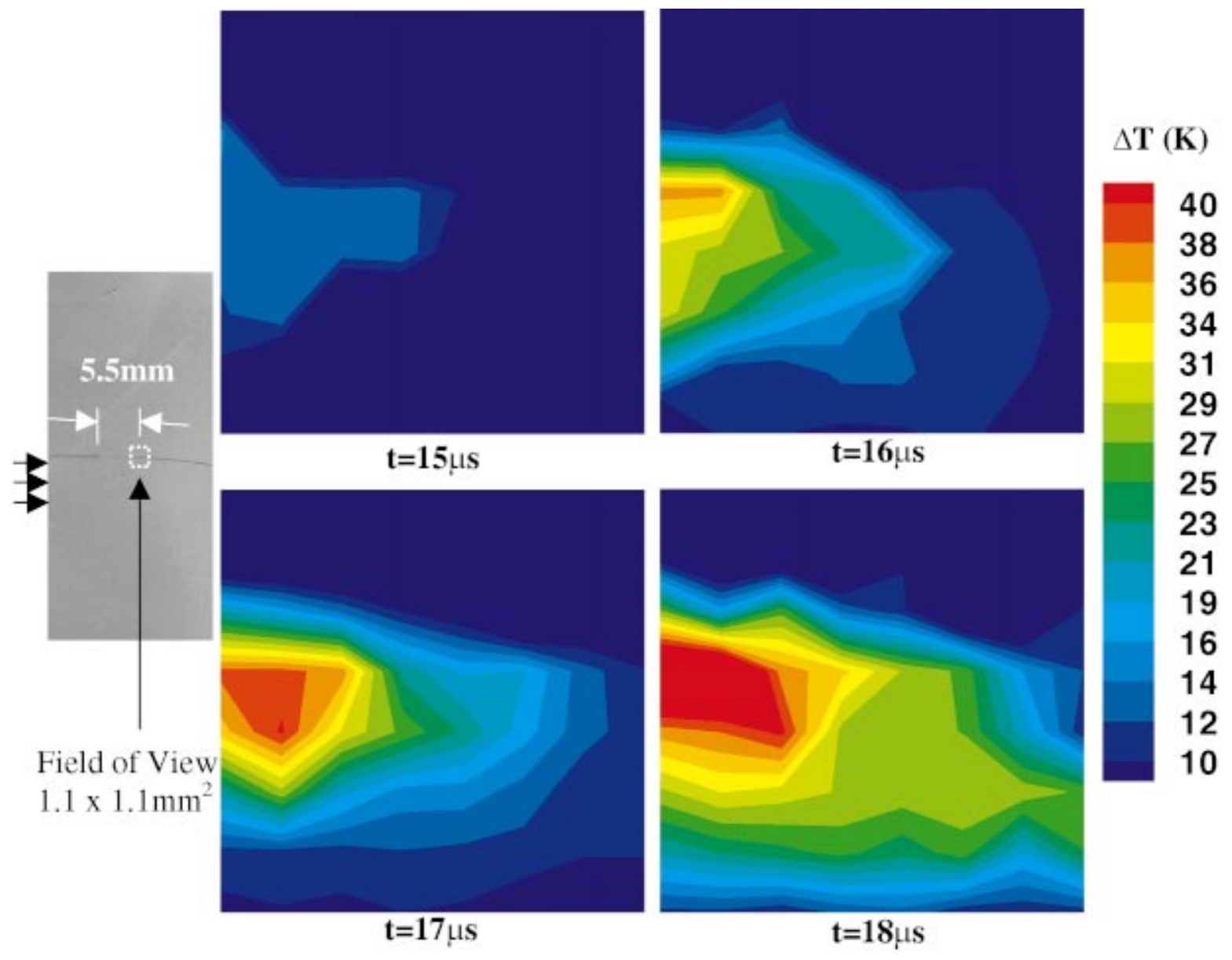

FIG. 5. (Color) A sequence of high-speed thermal images of an area that was $3 \mathrm{~mm}$ ahead of the fatigued crack tip. The images capture the thermal images of the shear band tip as it traverses across the field of view. These images, for the first time, establish length scales of the geometry of the shear band tip and the characteristic temperature gradient in an advancing shear band tip, which have only been a subject of conjecture until now.

front of the band where the material undergoes a rapid transition from a state of homogeneous deformation to a state of localized deformation. These images also reveal the length of the process zone to be about $500 \mu \mathrm{m}$ and the temperature gradient in it to be around $5 \times 10^{4} \mathrm{~K} / \mathrm{m}$. The order of magnitude of the length of the process zone is consistent with the predictions of Grady [12].

Perhaps the most surprising and significant finding of this investigation is the occurrence of a highly nonuniform temperature distribution along the length of the shear bands. As time progressed, the temperature distribution along the shear band was found to develop a highly transient, short range spatial periodicity in the direction of its growth. Figure 6 illustrates these intense temperature concentration regions that look like hot spots, which move along the band in time. Maximum temperatures shown are about $900 \mathrm{~K}$. This observation raises questions about the spatial and temporal nature of shear deformation inside a shear band, which until now has been assumed to be essentially laminar in all available theoretical models [12-15]. The high-speed images obtained here show the shearing process to be far from laminar and the hot spots observed indicate a possible instability operating at the bandwidth length scale, similar to certain hydro-

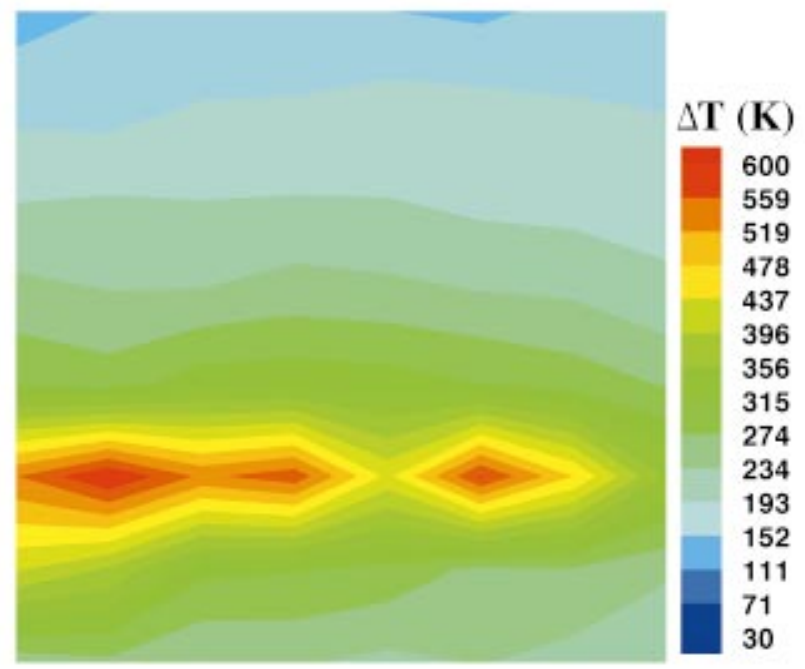

FIG. 6. (Color) A high-speed thermal image of a developed shear band whose tip is well outside the field of view, to the right. The area shown is $1.1 \times 1.1 \mathrm{~mm}^{2}$. Discrete hot spots were observed quite unexpectedly, suggesting an instability of laminar flow of the material within the shear band. 
dynamic instabilities observed in fluids. Since in a shear band a very thin layer of high temperature material is subjected to very high shear strain rates, the situation may resemble that of a viscous fluid confined between two rigid plates moving parallel to each other, known as the plane Couette flow in the fluid dynamics parlance. Since the plane Couette flow could become unstable and turbulent under certain conditions, we conjecture that the hot spots observed in the current experiments actually indicate certain instabilities of the deformation process. This led us to examine the stability characteristics of the plane Couette flow.

It is well known in fluid dynamics that plane Couette flow of inviscid as well as viscous fluids is linearly stable. However, the situation is different if the viscosity of the fluid is a function of temperature since viscous dissipation increases local temperature. The linear stability of plane Couette flow with viscous heating was numerically studied by Sukanek, Goldstein, and Laurence [22] and Yueh \& Weng [23]. They found that perturbations of finite wave numbers along the flow direction grow unboundedly beyond a critical Reynolds number, leading to eventual turbulence. This lends credence to our notion of the analogy with a flow related instability in a shear band since the temperature rise in a shear band decreases local viscosity of the material. Yet, there are major differences between a shear band and the Couette flow with viscous heating. The shear band temperatures are approximately one-half of the melting temperature of steel, indicating that the local shear band behavior cannot be necessarily approximated by that of a viscous fluid. Indeed, a more appropriate description of the material behavior would be one of a thermoviscoplastic material.

Spatially repeated nonuniform structures in sheared regions are also observed at an entirely different length scale in the earth's lower crust and upper mantle. Deep seismic reflection surveys have revealed the existence of lenticular shaped structures distributed within the shear zones. These structures are believed to be relatively less deformed regions surrounded by highly deformed material $[24,25]$. It has been proposed that these patterns influence the development of sedimentary basins, providing initiation sites for faults. Motivated by such observations, Molinari and Leroy [26] conducted a two-dimensional perturbation analysis of a layer of thermoviscoplastic material subjected to shear stress at the boundaries. The viscosity of the material they modeled was an exponentially decreasing function of temperature. They investigated the possibility of the existence of any perturbations with finite wave numbers in the direction along the band that could become unstable and grow as the deformation proceeds. They found that for certain material properties, such perturbations do exist and they lead to periodic hot spots along the length of the shear band. Their results, although quasistatic, can also be used to understand the present case of a shear band since the material used in our investigation has properties that are close to those of the material used in their analysis. The results of Molinari and Leroy [26] show that the nondimensional wave numbers of unstable perturbations range from $0.02-0.1$, which, using a shear band width of $40 \mu \mathrm{m}$ [Fig. 1(c)], would mean wavelengths of about 1.2-6.0 $\mathrm{mm}$. In our experiment, the distance between the hot spots was seen to be approximately $300 \mu \mathrm{m}$ (Fig. 6), which is remarkably close to the range predicted by their analysis, supporting our hypothesis that the observed nonuniform features are related to an instability of laminar flow of the material within the shear band. This finding calls for models that account for such vortical microstructures in order to accurately estimate the energy dissipation in shear bands and to describe the dynamics of shear band formation and growth. Motivated by the current experimental observations, detailed numerical simulations are currently underway using meshless schemes [27].

Our study has been able to establish the two-dimensional nature of temperature fields in regions of highly localized shear under transient dynamic loading conditions. A close examination of the temporal and spatial structure of the shear bands reveals the vortical nature of the temperature fields at the microscale. Such fresh insights into the deformation and failure of solids have been made possible by the newly developed high-speed IR microscopic camera, with the highest possible temporal resolution to date. Theories that could predict the formation of such instabilities would be able to capture the nature of energy dissipation within a shear band as well as the kinetics of shear band growth more accurately. We also believe that further investigations of the "hot-spot" structure observed in our experiments could lead to better understanding of similar nonuniform spatial structures surmised at geological length scales.

This work was supported by the Office of Naval Research Grant No. N00014-95-1-0453 (Dr. Y. D. S. Rajapakse, project manager) and the National Science Foundation Center for Quantitative Visualization at Caltech (Professor M. Gharib, Director). We are also pleased to acknowledge the contributions of Professor A. T. Zehnder, Cornell University for his pivotal role in developing the high-speed IR camera.
[1] R. J. Clifton, National Materials Advisory Board Report No. NMAB-356 (unpublished).

[2] P. Rosakis, A. J. Rosakis, G. Ravichandran, and J. Hodowany, J. Mech. Phys. Solids 48, 581 (2000).

[3] M. A. Meyers, Dynamic Behavior of Materials (Wiley, New York, 1994).

[4] J. P. Poirier, J. Struct. Geol. 2, 135 (1980).

[5] J. P. Brun and P. R. Cobbold, J. Struct. Geol. 2, 149 (1980).

[6] B. Evans and T. Wong, in Mechanics of Geomaterials, edited by Z. Bazant (Wiley, New York, 1985).

[7] R. J. Clifton, J. Duffy, K. A. Hartley, and T. G. Shawki, Scr. Metall. 18, 443 (1984).

[8] Y. L. Bai, J. Mech. Phys. Solids 30, 195 (1982).

[9] A. Molinari and R. J. Clifton, J. Appl. Mech. 54, 806 (1987).

[10] L. B. Freund, F. H. Wu, and M. Toulios, in Proceedings of the Considere Memorial Symposium, Paris, 1985, edited by J. Salencon (Presse de l'Ecole Nationale des Ponts et Chaussees, Paris, 1985). 
[11] S. Mercier and A. Molinari, J. Mech. Phys. Solids 46, 1463 (1998).

[12] D. E. Grady, J. Mech. Phys. Solids 40, 1197 (1992).

[13] T. W. Wright and G. Ravichandran, Int. J. Plast. 13, 309 (1997)

[14] A. Molinari and Y. M. Leroy, C. R. Acad. Sci., Ser. II: Mec., Phys., Chim., Sci. Terre Univers 310, 1017 (1990).

[15] G. Gioia and M. Ortiz, J. Mech. Phys. Solids 44, 251 (1996).

[16] A. Marchand and J. Duffy, J. Mech. Phys. Solids 36, 251 (1988)

[17] M. Zhou, A. J. Rosakis, and G. Ravichandran, J. Mech. Phys. Solids 44, 981 (1996).

[18] A. T. Zehnder, P. R. Guduru, A. J. Rosakis, and G. Ravichandran, Rev. Sci. Instrum. 71, 3762 (2000).
[19] J. F. Kalthoff, Appl. Mech. Rev. 43, S247 (1990).

[20] K. Ravi-Chandar, Int. J. Solids Struct. 32, 925 (1995).

[21] D. Rittel, Mech. Mater. 30, 217 (1998).

[22] P. C. Sukanek, C. A. Goldstein, and R. L. Laurence, J. Fluid Mech. 57, 651 (1973).

[23] C-S. Yueh and C-I. Weng, Phys. Fluids 8, 1802 (1996).

[24] D. J. Blundell, T. J. Reston, and A. M. Stein, Geophys. Monogr. 48, 57 (1989).

[25] A. M. Stein and D. J. Blundell, Tectonophysics 173, 455 (1990).

[26] A. Molinari and Y. M. Leroy, C. R. Acad. Sci., Ser. II: Mec., Phys., Chim., Sci. Terre Univers 313, 7 (1991).

[27] S. Li, W-K. Liu, W. Hao, T. Belytschko, and A. J. Rosakis (private communication). 